\title{
Good families of Drinfeld modular curves
}

\author{
Alp Bassa, Peter Beelen and Nhut Nguyen
}

\begin{abstract}
In this paper, we investigate examples of good and optimal Drinfeld modular towers of function fields. Surprisingly, the optimality of these towers has not been investigated in full detail in the literature. We also give an algorithmic approach for obtaining explicit defining equations for some of these towers and, in particular, give a new explicit example of an optimal tower over a quadratic finite field.
\end{abstract}

\section{Introduction}

Let $\mathbb{F}_{q}$ be a finite field with $q$ elements. For any absolutely irreducible, non-singular (projective) algebraic curve $X$ defined over $\mathbb{F}_{q}$ the genus $g(X)$ and the number of rational points $N_{1}(X)$ satisfy the inequality $N_{1}(X) \leqslant q+1+2 \sqrt{q} g(X)$. This inequality is known as the Hasse-Weil bound. To investigate the asymptotic behaviour of such curves with increasing genus, Ihara introduced the quantity

$$
A(q):=\limsup _{g(X) \rightarrow \infty} \frac{N_{1}(X)}{g(X)}
$$

where the limit is over all projective, absolutely irreducible, non-singular algebraic curves defined over $\mathbb{F}_{q}$. It is known that $0<A(q) \leqslant \sqrt{q}-1$, the first inequality being due to Serre [14], while the second inequality is known as the Drinfeld-Vlâdut bound [17]. Combining the work of Ihara [12] and the Drinfeld-Vladut bound, one sees that $A(q)=\sqrt{q}-1$ if $q$ is a square. Note that, for non-square values of $q$, the true value of $A(q)$ is currently unknown.

There exist a variety of constructions showing that $A(q)=\sqrt{q}-1$ if $q$ is a square. By the Drinfeld-Vladut bound, it is sufficient to show that $A(q) \geqslant \sqrt{q}-1$, in this case. Ihara used families of Shimura modular curves for this purpose [12], while Tsfasman et al. used families of (classical) modular curves (for $q=p^{2}$ and $q=p^{4}$ ) [16]. Gekeler showed that certain families of Drinfeld modular curves can also be used [8]. A different and completely explicit approach was presented by Garcia and Stichtenoth [4]. For any square $q$, they presented an explicitly defined family of curves $C_{i}$ (or rather towers of function fields $\left(F_{i}\right)_{i}$ ), defined over $\mathbb{F}_{q}$, for which the ratio $N_{1}\left(F_{i}\right) / g\left(F_{i}\right)$ tends to $\sqrt{q}-1$. Such families are called asymptotically optimal. This discovery led to an alternative approach for obtaining lower bounds on $A(q)$ and, by the explicit nature of their construction, the resulting function fields are more apt for applications in, for example, the theory of error-correcting codes $[\mathbf{1 0}, \mathbf{1 6}]$. Despite the apparent difference between this approach and the constructions given in [4], it was shown by Elkies that the same equations can be obtained using Drinfeld modular curves [3]. Conversely, the theory of modular curves can be used to produce explicitly defined families of curves $[\mathbf{1}, \mathbf{2}]$. The current work can be seen as a continuation and solidification of the work started in [1] to explicitly define families of Drinfeld modular curves. We will, on occasion, use the language of function fields rather than the more geometric language of curves to describe such families.

Received 25 November 2014; revised 26 June 2015.

2010 Mathematics Subject Classification 11G09, 14H05, 14H10 (primary), 11R58, 14Q05 (secondary). 


\section{Preliminaries}

To put this work into the right context of Drinfeld modular curves, we briefly recall some notions that we will use in the remainder of the paper. See $[6]$ for a more detailed exposition on Drinfeld modular curves and [11] for an exposition on Drinfeld modules. Let $F / \mathbb{F}_{q}$ be a function field with full constant field $\mathbb{F}_{q}$ and let $P$ be a place of degree $d$. Then we denote by $F_{P}$ the residue field of $P$. It is a finite field with $\left|F_{P}\right|:=q^{d}$ elements. For an integer $e \geqslant 1$, we denote by $F_{P}^{(e)}$ the algebraic extension of $F_{P}$ of degree $e$. In the theory of Drinfeld modules and Drinfeld modular curves, one singles out a place $P_{\infty}$ of $F$ (playing the role of a place at 'infinity') and defines the ring $A$ as the ring of all functions in $F$ that are regular outside $P_{\infty}$. We will denote the degree of $P_{\infty}$ by $\delta$. Note that prime ideals of $A$ can be identified with places of $F$ distinct from $P_{\infty}$. For an ideal $\mathfrak{n} \subset A$ we define $|\mathfrak{n}|:=|A / \mathfrak{n}|$ and $\operatorname{deg} \mathfrak{n}:=\log _{q}|\mathfrak{n}|$. In the case where $\mathfrak{n}=(a)$ is a principal ideal, we write $\operatorname{deg} a:=\operatorname{deg}(a)$. In the special case of $F=\mathbb{F}_{q}(T)$ and $P_{\infty}$ being the pole of $T$, one gets $\delta=1$ and $A=\mathbb{F}_{q}[T]$. In this case, places of $F$ different from $P_{\infty}$ can be identified with monic irreducible polynomials and ideals of $A$ with monic polynomials.

Let $L$ be a field and $\iota: A \rightarrow L$ a homomorphism. The kernel of $\iota$ is called the $A$ characteristic of $L$. Let $L\{\tau\}$ be the non-commutative polynomial ring generated by the Frobenius endomorphism $\tau$ satisfying $\tau r=r^{q} \tau$ for all $r \in L$. Then an A-Drinfeld module over $L$ of rank 2 is a homomorphism

$$
\begin{aligned}
\phi: A & \rightarrow L\{\tau\} \\
a & \mapsto \phi_{a}
\end{aligned}
$$

such that for all $a \in A \backslash\{0\}$, we have $\operatorname{deg}_{\tau} \phi_{a}=2 \operatorname{deg} a$, and the constant term of $\phi_{a}$ is equal to $\iota(a)$. Elements of $L\{\tau\}$ can also be interpreted as linearized polynomials by replacing $\tau^{i}$ by $X^{q^{i}}$. This makes it possible to evaluate elements of $L\{\tau\}$ at elements of $\bar{L}$, the algebraic closure of $L$. Let $\mathfrak{n} \subset A$ be an ideal of $A$, then we define $\phi[\mathfrak{n}]$ to be the set of elements $x \in \bar{L}$ such that $\phi_{a}(x)=0$ for all $a \in \mathfrak{n}$. This set is called the set of $\mathfrak{n}$-torsion points of the Drinfeld module $\phi$. If $\mathfrak{n}$ is coprime with the $A$-characteristic of $L$, then $\phi[\mathfrak{n}] \cong(A / \mathfrak{n})^{2}$ as an $A$-module. Two Drinfeld modules $\phi$ and $\psi$ with the same $A$-characteristic are called isogenous if there exists $\lambda \in L\{\tau\}$ different from zero such that $\lambda \phi_{a}=\psi_{a} \lambda$ for all $a \in A$. The element $\lambda$ is called an isogeny. The Drinfeld modules $\phi$ and $\psi$ are called isomorphic if $\lambda$ can be chosen from $\bar{L} \backslash\{0\}$. If the kernel of the isogeny $\lambda$ is a free $A / \mathfrak{n}$ module of rank one contained in $\phi[\mathfrak{n}]$, then $\lambda$ is called an $\mathfrak{n}$-isogeny.

For a non-zero monic polynomial $\mathfrak{n} \in \mathbb{F}_{q}[T]$ Gekeler investigates in [5] (among other things) the Drinfeld modular curve $Y_{0}(\mathfrak{n})$. The points on this curve parametrize isomorphism classes of pairs of $\mathbb{F}_{q}[T]$-Drinfeld modules of rank two together with an $\mathfrak{n}$-isogeny between them. Adding so-called cusps gives a projective algebraic curve $X_{0}(\mathfrak{n})$ defined over $F$ that, in general, however, will not be absolutely irreducible. In the case where $\mathfrak{n}=1$, the number of cusps is seen to be $(\delta \cdot h(F))^{2}$ while $X_{0}(1)$ has $\delta \cdot h(F)$ components [6, VI.5]. Here $h(F)$ denotes the class number of the function field $F$. This implies that the number of absolutely irreducible components of $X_{0}(\mathfrak{n})$ equals $\delta \cdot h(F)$. Equivalently, the number of components is equal to $h(A)$, the cardinality of the ideal class group of the ring $A$. By considering the action of the ideal class group of $A$, one sees that the cusps are distributed equally among the absolutely irreducible components of $X_{0}(1)$, which implies that any such component contains exactly $\delta \cdot h(F)$ cusps. We will denote by $x_{0}(\mathfrak{n})$ an absolutely irreducible component of $X_{0}(\mathfrak{n})$. For any prime ideal of $A$ (corresponding to a place of $F$ different from $P_{\infty}$ ), one obtains, by reduction, an algebraic curve defined over a finite field. In the case of $A=\mathbb{F}_{q}[T]$ and $\delta=1$, the curve $X_{0}(\mathfrak{n}$ ) (as well as its reduction modulo any prime $P$ relatively prime to $\mathfrak{n}$ ) is absolutely irreducible. By computing the precise formula for the genus and the number of rational points on reductions of $\mathbb{F}_{q}[T]$ Drinfeld modular curves $X_{0}(\mathfrak{n})$, Gekeler [8] showed that, for a series $\left(\mathfrak{n}_{k}\right)_{k \in \mathbb{N}}$ of polynomials of 
$A$ coprime with an irreducible polynomial $P \in A$, with degrees that tend to infinity, the family of Drinfeld modular curves $X_{0}\left(\mathfrak{n}_{k}\right) / F_{P}$ attains the Drinfeld-Vladut bound when considered over $F_{P}^{(2)}$. In the case where $\mathfrak{n}_{k}=T^{k}$ and $P=T-1$, explicit equations for the modular curves $X_{0}\left(T^{k}\right)$ were given in [2], while some more general examples (including defining equations in generic $A$-characteristic 0 ) were given in [1]. For $A=\mathbb{F}_{q}[T]$ and $\delta=1$ the situation has therefore, to a large extent, been investigated both theoretically and explicitly. However, we will see that generalizations to other rings $A$ and values of $\delta$ are possible and that, in some cases, the resulting families of curves can be described by equations explicitly.

\section{Genus calculation of $x_{0}(\mathfrak{n})$}

In this section we will compute the genus of (an irreducible component of) the modular curve $X_{0}(\mathfrak{n})$. We put no restriction on the choice of function field $F$ and place $P_{\infty}$. A recipe for this genus computation is given in [6] using results from [5]. The recipe was carried out in [6] in the case where $\mathfrak{n}$ is a prime ideal. We will, in this section, carry out the computations for any ideal $\mathfrak{n}$. The computations in $[\mathbf{5}, \mathbf{6}]$ are carried out over the field $C_{\infty}$, which is the completion of the algebraic closure of the completion of $F$ at $P_{\infty}$. For our purposes, one therefore needs to check that the genus of $x_{0}(\mathfrak{n})$ does not change when changing the constant field. For $A=\mathbb{F}_{q}[T]$, this result is contained in [13]. In our case, note that the only points that ramify in the cover $X(\mathfrak{n}) / X(1)$ are the elliptic points of $X(1)$ and the cusps of $X(1)$. The residue field of a cusp is isomorphic to the Hilbert class field of $F$ [6, Theorem 1.9(ii), p. 81], while the residue field of an elliptic point is a subfield of the Hilbert class field of $\mathbb{F}_{q^{2}} F$ [6, Proposition 2.2, p. 83]. In either case, the residue field is a separable extension of the field $F$. Using [9, Corollary 3.4.2], we see that the argument given in [13] carries over to our situation.

One of the ingredients in the genus expressions of $x_{0}(\mathfrak{n})$ involve the L-polynomial of the function field $F$, which we will denote by $P(t)$. Note that $P(1)=h(F)$, which is the class number of $F$. The following functions will also be useful.

Definition 1. Let $\mathfrak{n} \subset A$ be an ideal and suppose that $\mathfrak{n}=\mathfrak{p}_{1}^{r_{1}} \ldots \mathfrak{p}_{s}^{r_{s}}$, for prime ideals $\mathfrak{p}_{1}, \ldots, \mathfrak{p}_{s}$ and positive integers $r_{1}, \ldots, r_{s}$. Writing $q_{i}:=\left|\mathfrak{p}_{i}\right|$, we define

$$
\begin{gathered}
\varphi(\mathfrak{n}):=\left|(A / \mathfrak{n})^{*}\right|=\prod_{i=1}^{s} q_{i}^{r_{i}-1}\left(q_{i}-1\right), \\
\varepsilon(\mathfrak{n}):=\prod_{i=1}^{s} q_{i}^{r_{i}-1}\left(q_{i}+1\right)
\end{gathered}
$$

and

$$
\kappa(\mathfrak{n}):=\prod_{i=1}^{s}\left(q_{i}^{\left[r_{i} / 2\right]}+q_{i}^{r_{i}-\left[r_{i} / 2\right]-1}\right),
$$

where $[r]$ denotes the integral part of a real number $r$.

Using these notions, we will obtain the following theorem.

Theorem 3.1. Let $A$ and $\mathfrak{n}$ be as above. In particular, suppose that $\mathfrak{n}=\mathfrak{p}_{1}^{r_{1}} \ldots \mathfrak{p}_{s}^{r_{s}}$, for prime ideals $\mathfrak{p}_{1}, \ldots, \mathfrak{p}_{s}$ and positive integers $r_{1}, \ldots, r_{s}$. Then

$$
g\left(x_{0}(\mathfrak{n})\right)=1+\frac{\left(q^{\delta}-1\right) \varepsilon(\mathfrak{n}) P(q)}{\left(q^{2}-1\right)(q-1)}-\frac{P(1) \delta\left(\kappa(\mathfrak{n})+2^{s-1}(q-2)\right)}{q-1}+\eta,
$$

where $\eta=-P(-1) 2^{s-1} q /(q+1)$ if $\delta$ is odd and all prime divisors of $\mathfrak{n}$ are of even degree; and $\eta=0$ otherwise. 
Note that [6, VII. 5.13] (the case where $\mathfrak{n}$ is a prime ideal) is a special case of this theorem.

The recipe outlined in [6] consists of the following ingredients: first compute the genus of $x_{0}(1)$, then consider the cover $x_{0}(\mathfrak{n}) / x_{0}(1)$. Since, in general (as in the case of classical modular curves) this cover is not Galois, one studies a Galois cover $x(\mathfrak{n}) / x_{0}(1)$ first. The curve $x(\mathfrak{n})$ is an irreducible component of the modular curve $X(\mathfrak{n})$, whose points correspond to isomorphism classes of $A$-Drinfeld modules $\phi$ of rank two together with an isomorphism of $\phi[\mathfrak{n}]$ with $(A / \mathfrak{n})^{2}$. Note that $X_{0}(1)=X(1)$ and that the points on this curve correspond to isomorphism classes of $A$-Drinfeld modules of rank two.

Since $x(\mathfrak{n}) / x(1)$ is Galois, so is $x(\mathfrak{n}) / x_{0}(\mathfrak{n})$. The Galois group of the cover $x(\mathfrak{n}) / x(1)$ (respectively, $x(\mathfrak{n}) / x_{0}(\mathfrak{n})$ ), is given by $G(\mathfrak{n})$ (respectively, $H(\mathfrak{n})$ ) defined as [6, VII.5]

$$
G(\mathfrak{n}):=\left\{\gamma \in \operatorname{GL}(2, A / \mathfrak{n}): \operatorname{det} \gamma \in \mathbb{F}_{q}^{*}\right\} / Z\left(\mathbb{F}_{q}\right)
$$

and

$$
H(\mathfrak{n}):=\left\{\left(\begin{array}{ll}
a & b \\
0 & d
\end{array}\right) \in \mathrm{GL}(2, A / \mathfrak{n}): a d \in \mathbb{F}_{q}^{*}\right\} / Z\left(\mathbb{F}_{q}\right)
$$

with

$$
Z\left(\mathbb{F}_{q}\right):=\left\{\left(\begin{array}{cc}
a & 0 \\
0 & a
\end{array}\right): a \in \mathbb{F}_{q}^{*}\right\}
$$

Before proceeding, we calculate the cardinalities of the groups $G(\mathfrak{n})$ and $H(\mathfrak{n})$. The latter cardinality is relatively easy since, in that case, $a \in(A / \mathfrak{n})^{*}$ and $b \in A / \mathfrak{n}$ can be chosen freely (leaving $q-1$ possibilities for $d$ ). Therefore,

$$
|H(\mathfrak{n})|=\left|(A / \mathfrak{n})^{*}\right| \cdot(q-1) \cdot|A / \mathfrak{n}| /(q-1)=\varphi(\mathfrak{n})|\mathfrak{n}| .
$$

To count the cardinality of $G(\mathfrak{n})$, observe that

$$
|\mathrm{SL}(2, A / \mathfrak{n})|=\frac{\left|\left\{\gamma \in \mathrm{GL}(2, A / \mathfrak{n}): \operatorname{det} \gamma \in \mathbb{F}_{q}^{*}\right\}\right|}{q-1},
$$

since any non-zero value in $\mathbb{F}_{q}$ of the determinant is taken equally often when considering elements in $\left\{\gamma \in \mathrm{GL}(2, A / \mathfrak{n}): \operatorname{det} \gamma \in \mathbb{F}_{q}^{*}\right\}$. By definition of $G(\mathfrak{n})$, we obtain that

$$
|G(\mathfrak{n})|=|\mathrm{SL}(2, A / \mathfrak{n})| .
$$

The cardinality of $\operatorname{SL}(2, A / \mathfrak{n})$ is well known and can be computed using the Chinese remainder theorem. This approach gives that if $\mathfrak{n}=\prod_{i} \mathfrak{p}_{i}^{r_{i}}$ for prime ideals $\mathfrak{p}_{i} \subset A$, then

$$
|\mathrm{SL}(2, A / \mathfrak{n})|=\prod_{i}\left|\mathrm{SL}\left(2, A / \mathfrak{p}_{i}^{r_{i}}\right)\right|=\prod_{i}\left|\mathfrak{p}_{i}\right|^{3 r_{i}-2}\left(\left|\mathfrak{p}_{i}\right|^{2}-1\right)=\varphi(\mathfrak{n}) \varepsilon(\mathfrak{n})|\mathfrak{n}|,
$$

implying that

$$
|G(\mathfrak{n})|=\varphi(\mathfrak{n}) \varepsilon(\mathfrak{n})|\mathfrak{n}| .
$$

We now turn our attention again to the Galois cover $x(\mathfrak{n}) / x(1)$. It was shown in $[6]$ that the only ramification in this cover occurs above the so-called elliptic points (with ramification index $q+1$ ) and the cusps of $x(1)$. Moreover, as mentioned before, the number of cusps on $x(1)$ equals $\delta h(F)$. The elliptic points were studied in [6, V.4, VII.5]: the number of elliptic points on $x(1)$ is zero if $\delta$ is even and $P(-1)$ if $\delta$ is odd, each having ramification index $q+1$ in the cover $x(\mathfrak{n}) / x(1)$. We now write $\mathfrak{n}=\mathfrak{p}_{1}^{r_{1}} \ldots \mathfrak{p}_{s}^{r_{s}}$ for prime ideals $\mathfrak{p}_{1}, \ldots, \mathfrak{p}_{s}$ of $A$ and positive integers $r_{1}, \ldots, r_{s}$, just as before. Although $x(1)$ contains $P(-1)$ elliptic points if $\delta$ is odd, such an elliptic point does not give rise to ramification in the cover $x(\mathfrak{n}) / x_{0}(\mathfrak{n})$ if any of the $\mathfrak{p}_{i}$ has odd degree. If $\delta$ is odd and all prime ideals $\mathfrak{p}_{i}$ occurring in the decomposition of $\mathfrak{n}$ have even 
degree, among all the points of $x_{0}(\mathfrak{n})$ that are lying above a given elliptic point of $x(1)$ there are exactly $2^{s}$ that are ramified in the covering $x(\mathfrak{n}) / x_{0}(\mathfrak{n}$ ) (with ramification index $q+1$ ). This completely determines the behaviour of elliptic points as far as their role in the genus computation of $x(\mathfrak{n})$ and $x_{0}(\mathfrak{n})$ is concerned. To describe the behaviour of the cusps, we start by describing their ramification groups in $x(\mathfrak{n}) / x(1)$ (following [6, VII.5]).

Lemma 3.2 [6, Lemma 5.6]. Let

$$
G(\mathfrak{n})_{\infty}:=\left\{\left(\begin{array}{ll}
a & b \\
0 & d
\end{array}\right) \in \operatorname{GL}(2, A / \mathfrak{n}): a, d \in \mathbb{F}_{q}^{*}\right\} / Z\left(\mathbb{F}_{q}\right) .
$$

Then the stabilizers of all cusps of $x(\mathfrak{n})$ are conjugate in $G(\mathfrak{n})$ to $G(\mathfrak{n})_{\infty}$.

This means, in particular, that the ramification index in $x(\mathfrak{n}) / x(1)$ of any cusp equals $\left|G(\mathfrak{n})_{\infty}\right|=(q-1)^{2}|\mathfrak{n}| /(q-1)=(q-1)|\mathfrak{n}|$. The cardinality of the first (respectively, second) ramification group of any cusp is then calculated in [6, Lemma 5.7] to be $|\mathfrak{n}|$ (respectively, one). This means that the different exponent for a cusp equals $(q-1)|\mathfrak{n}|-1+|\mathfrak{n}|-1=$ $q|\mathfrak{n}|-2$. Combining this information concerning the ramification groups of the cusps with the description of the ramification behaviour of the elliptic points makes the computation of the genus of $x(\mathfrak{n})$ completely feasible using the Riemann-Hurwitz genus formula. The result (given in slightly less explicit form in [6, Theorem 5.11]) is

$$
g(x(\mathfrak{n}))=1+\frac{\left(q^{\delta}-1\right) P(q)}{\left(q^{2}-1\right)(q-1)} \varphi(\mathfrak{n}) \varepsilon(\mathfrak{n})|\mathfrak{n}|-\frac{\delta P(1)}{q-1} \varphi(\mathfrak{n}) \varepsilon(\mathfrak{n}) .
$$

The ramification behaviour of the cusps is more complicated in the cover $x(\mathfrak{n}) / x_{0}(\mathfrak{n})$. However, in [6, VII.5] (with reference to [5, 3.4.15]) the total contribution to the RiemannHurwitz genus formula for the cover $x(\mathfrak{n}) / x_{0}(\mathfrak{n})$ of all cusps of $x(\mathfrak{n})$ lying above a single cusp of $x(1)$ is computed to be

$$
(q-1)^{-1} \varphi(\mathfrak{n})\left(2|\mathfrak{n}| \kappa(\mathfrak{n})+2^{s}(q-2)|\mathfrak{n}|-2 \varepsilon(\mathfrak{n})\right) .
$$

We now have all the ingredients needed for the proof of Theorem 3.1

Proof. For any point $P$ of $x(\mathfrak{n})$, we denote by $e(P)$ (respectively, $d(P)$ ) the ramification index (respectively, different exponent) in the cover $x(\mathfrak{n}) / x_{0}(\mathfrak{n})$. Since the only ramified points in the cover $x(\mathfrak{n}) / x(1)$ are the cusps and the elliptic points (if these exist), by applying the Riemann-Hurwitz genus formula for the cover $x(\mathfrak{n}) / x_{0}(\mathfrak{n})$ we obtain

$$
2 g(x(\mathfrak{n}))-2=\varphi(\mathfrak{n})|\mathfrak{n}|\left(2 g\left(x_{0}(\mathfrak{n})\right)-2\right)+\sum_{P \text { cusp }} d(P)+\sum_{\begin{array}{c}
P \text { elliptic } \\
\text { point }
\end{array}} d(P) .
$$

The sum concerning the elliptic points is zero if no such points exist and therefore

$$
\sum_{\substack{\text { elliptic } \\ \text { point }}} d(P)=0
$$

if $\delta$ is even or if there exists $\mathfrak{p}_{i}$ of odd degree. Otherwise, as we have described previously, above each of the $P(-1)$ cusps of $x(1)$ lie exactly $2^{s}$ points of $x_{0}(\mathfrak{n})$ that ramify with ramification index $q+1$ in $x(\mathfrak{n}) / x_{0}(\mathfrak{n})$. This implies that

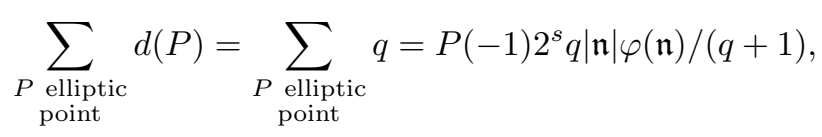

if $\delta$ is odd and all prime divisors of $\mathfrak{n}$ have even degree. 
The summation over the points lying over any of the $\delta h(F)$ cusps of $x(1)$ can be dealt with using equation (3.4). We obtain that

$$
\sum_{P \text { cusp }} d(P)=\delta h(F)(q-1)^{-1} \varphi(\mathfrak{n})\left(2|\mathfrak{n}| \kappa(\mathfrak{n})+2^{s}(q-2)|\mathfrak{n}|-2 \varepsilon(\mathfrak{n})\right) .
$$

Substituting these values into equation (3.5) and using equation (3.3), Theorem 3.1 follows.

\section{Rational points on reductions of Drinfeld modular curves}

In this section, we combine the previously described genus computation of the curves $x_{0}(\mathfrak{n})$ with the fact that reductions of these curves have many rational points (when the field of definition is chosen properly). We will show that, for any sequence of ideals $\left(\mathfrak{n}_{k}\right)_{k \geqslant 1}$ such that $\operatorname{deg} \mathfrak{n}_{k} \rightarrow \infty$ as $k \rightarrow \infty$, the corresponding family of reductions of Drinfeld modular curves $\left(x_{0}\left(\mathfrak{n}_{k}\right)\right)_{k}$ has good asymptotic properties. In [15], the (reductions of the) curves $x_{0}(\mathfrak{n})$ were also investigated in the case where $\mathfrak{n}$ is a principal ideal, using a different method inspired by [12]. Our approach is to use, for any ideal $\mathfrak{n}$, results from [7] to estimate the number of rational points on the reduction of $x_{0}(\mathfrak{n})$ and to use the explicit genus formula for $g\left(x_{0}(\mathfrak{n})\right)$ from the previous section.

While the curves $X_{0}(\mathfrak{n})$ themselves are defined over the function field $F$ (and a component $x_{0}(\mathfrak{n})$ over an extension field of $F$ ), a model can be found that can be reduced modulo prime ideals of the ring $A$. This reduction is known to be good if $P \subset A$ is a prime ideal which is coprime with the ideal $\mathfrak{n}$. Thus, reduction modulo $P$ gives rise to a curve (as before not necessarily absolutely irreducible) that is defined over the finite field $A / P$. For convenience, we write $F_{P}:=A / P$ and denote by $F_{P}^{(m)}$ the degree $m$ extension of $F_{P}$. In the where case $A=$ $\mathbb{F}_{q}[T]$, these reduced Drinfeld modular curves have many rational points over $F_{P}^{(2)}$ (essentially corresponding to supersingular $A$-Drinfeld modules), but it turns out that, in general, the situation is slightly more complicated. As a matter of fact, the supersingular Drinfeld modules in $A$-characteristic $P$ are,in general, defined over the field $F_{P}^{(2 e)}=\mathbb{F}_{q^{2 d e}}$ with $d=\operatorname{deg} P$ and $e=\operatorname{ord} P$, the order of the ideal $P$ in the ideal class group of the ring $A[\mathbf{7}, \S 4]$.

More precisely, in [7] it was shown that, for a prime ideal $P \subset A$ with $d:=\operatorname{deg} P$, the number $N(P)$ of isomorphism classes of supersingular $A$-Drinfeld modules in $A$-characteristic $P$ equals $N(P)=h_{1}(P)+h_{2}(P)$ with

$$
h_{1}(P):= \begin{cases}\delta P(1)\left(P(q) \frac{\left(q^{\delta}-1\right)\left(q^{d}-1\right)}{\left(q^{2}-1\right)(q-1)}-\frac{P(-1)}{q+1}\right) & \text { if } d \text { and } \delta \text { are odd } \\ \delta P(1) P(q) \frac{\left(q^{\delta}-1\right)\left(q^{d}-1\right)}{\left(q^{2}-1\right)(q-1)} & \text { otherwise }\end{cases}
$$

and

$$
h_{2}(P):= \begin{cases}\delta P(1) P(-1) & \text { if } d \text { and } \delta \text { are odd } \\ 0 & \text { otherwise }\end{cases}
$$

Each isomorphism class of a supersingular $A$-Drinfeld module gives rise to a rational point (which we will call a supersingular point) on the curve $X(1)$, if the field of definition is taken to be $F_{P}^{(2 e)}$. Using the action given by the class group of $A$ on the absolutely irreducible components of $X(1)$, one sees that the supersingular points are equidistributed among all $\delta P(1)$ components of $X(1)$. These observations enable us to give a lower bound on the number of rational points on $x_{0}(\mathfrak{n})$. 
Theorem 4.1. Let $\mathfrak{n} \subset A$ be an ideal prime to the $A$-characteristic $P$ and suppose that $\mathfrak{n}=\mathfrak{p}_{1}^{r_{1}} \ldots \mathfrak{p}_{s}^{r_{s}}$, for prime ideals $\mathfrak{p}_{1}, \ldots, \mathfrak{p}_{s}$ and positive integers $r_{1}, \ldots, r_{s}$. Moreover, denote $d:=\operatorname{deg} P$ and $e:=\operatorname{ord} P$. Consider a component $x_{0}(\mathfrak{n})$ of $X_{0}(\mathfrak{n})$ over the finite field $F_{P}^{(2 e)}$ and denote its number of rational points by $N_{1}\left(x_{0}(\mathfrak{n})\right)$. Then, if $d, \delta$ are odd and $\operatorname{deg} \mathfrak{p}_{i}$ is even for all $i$,

$$
N_{1}\left(x_{0}(\mathfrak{n})\right) \geqslant \varepsilon(\mathfrak{n}) P(q) \frac{\left(q^{\delta}-1\right)\left(q^{d}-1\right)}{\left(q^{2}-1\right)(q-1)}+P(-1) 2^{s} \frac{q}{q+1},
$$

while otherwise,

$$
N_{1}\left(x_{0}(\mathfrak{n})\right) \geqslant \varepsilon(\mathfrak{n}) P(q) \frac{\left(q^{\delta}-1\right)\left(q^{d}-1\right)}{\left(q^{2}-1\right)(q-1)} .
$$

Proof. All points of $x_{0}(\mathfrak{n})$ lying above one of the $N(P) /(\delta P(1))$ supersingular points of $x(1)$ are rational, but not necessarily unramified in the covering $x_{0}(\mathfrak{n}) / x(1)$. The reason for this is that the elliptic points are supersingular points if (and only if) both $\delta$ and $d$ are odd [7, Lemma 7.2]. However, any elliptic point has ramification index either one or $q+1$ in the cover $x_{0}(\mathfrak{n}) / x(1)$. Moreover, from [6, V.4, VII.5] we see that if $\delta$ is odd and all prime ideals $\mathfrak{p}_{i}$ occurring in the decomposition of $\mathfrak{n}$ have even degree, among all the points of $x_{0}(\mathfrak{n})$ that are lying above a given elliptic point of $x(1)$ there are exactly $2^{s}$ that are ramified in the covering $x(\mathfrak{n}) / x_{0}(\mathfrak{n})$ (with ramification index $q+1$ ). The latter statement is equivalent to saying that these $2^{s}$ points of $x_{0}(\mathfrak{n})$ have ramification index one in $x_{0}(\mathfrak{n}) / x(1)$. Counting the number of points of $x_{0}(\mathfrak{n})$ lying above the supersingular points of $x(1)$ is now direct and yields the stated lower bound on $N_{1}\left(x_{0}(\mathfrak{n})\right)$.

From Theorem 3.1 we get the following asymptotic result.

Theorem 4.2. Let $A$ be any ring of functions regular outside a fixed place $\infty$ of degree $\delta$. Let $P \subset A$ be a prime ideal of degree $d$ and order $e$ and, further, let $\left(\mathfrak{n}_{k}\right)_{k \geqslant 1}$ be a series of ideals relatively prime to $P$. The family of reductions of Drinfeld modular curves $\left(x_{0}\left(\mathfrak{n}_{k}\right)\right)_{k}$ when defined over $\mathbb{F}_{q^{2 d e}}$ satisfies

$$
\lim _{k \rightarrow \infty} \frac{N_{1}\left(x_{0}\left(\mathfrak{n}_{k}\right)\right)}{g\left(x_{0}\left(\mathfrak{n}_{k}\right)\right)} \geqslant q^{d}-1
$$

Remark 1. The lower bound given in Theorem 4.2 is sharp in the case where $P$ is a principal ideal, since, in this case, $e=1$ and the given lower bound is equal to the Drinfeld-Vladut upper bound. If $A=\mathbb{F}_{q}[T]$ (in particular $\delta=1$ ), the ideal class group of $A$ is trivial, implying that any family of reductions of Drinfeld modular curves, as in Theorem 4.2, has optimal asymptotic properties. This particular case was shown in [8]. If $P$ is not principal, the resulting families will be asymptotically good, but not optimal. Note that in [15] this subtlety is missing.

\section{A recursive description of a Drinfeld modular tower}

In this section we will illustrate Theorem 4.2 by describing some families of Drinfeld modular curves $\left(x_{0}\left(\mathfrak{n}_{k}\right)\right)_{k}$ more explicitly. In the case where $\mathfrak{n}_{k}=\mathfrak{p}^{k}$ for a fixed prime ideal $\mathfrak{p}$ of $A$, this can be done in a recursive way (in fact, $\mathfrak{p}$ could be any non-trivial ideal, but we will assume primality for simplicity). The reason for this is similar to the reasoning presented in $[\mathbf{2}, \mathbf{3}]$, but is somewhat more involved due to the fact that the curves $X(1)$ and $X_{0}\left(\mathfrak{p}^{k}\right)$ are not, in general, absolutely irreducible. Therefore, we go through the argument in the following.

A point on $X_{0}(\mathfrak{p})$ corresponds to an isomorphism class $[\phi, \psi]$ of a pair of $\mathfrak{p}$-isogenous $A$ Drinfeld modules of rank two. Therefore, there are two possible maps, say $\pi_{1}$ and $\pi_{2}$, from 


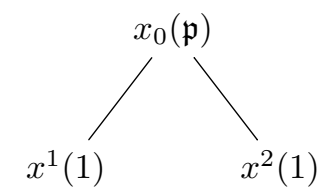

Figure 1. A correspondence of modular curves.

$X_{0}(\mathfrak{p})$ to $X(1)$ (see Figure 1), since one can send $[\phi, \psi]$ to $[\phi]$ or $[\psi]$ (the isomorphism class of $\phi$ or that of $\psi$ ). Since a $\mathfrak{p}$-isogeny corresponds to a cyclic submodule of the $\mathfrak{p}$-torsion points of $\phi$, the degree of the first map is $|\mathfrak{p}|+1$. By symmetry, the degree of the second map is also $|\mathfrak{p}|+1$.

The image of a fixed absolutely irreducible component $x_{0}(\mathfrak{p})$ of $X_{0}(\mathfrak{p})$ under either $\pi_{1}$ or $\pi_{2}$, will be an absolutely irreducible component of $X(1)$, but not necessarily the same one. We denote these components by $x^{1}(1)$ and $x^{2}(1)$. We can then view $x_{0}(\mathfrak{p})$ as a curve lying inside $x^{1}(1) \times x^{2}(1)$. Once an explicit description of the components of $x^{1}(1)$ and $x^{2}(1)$ is available, the map $\pi_{1} \times \pi_{2}: x_{0}(\mathfrak{p}) \rightarrow x^{1}(1) \times x^{2}(1)$, defined by $[\phi, \psi] \mapsto([\phi],[\psi])$, can be, in principle, be used to describe the curve $x_{0}(\mathfrak{p})$ explicitly by equations. However, in practice, it is very convenient to assume that the genera of the components of $X(1)$ are zero. In this case, a component $x^{i}(1)$ can just be described using a single variable $u_{i}$, which one can think of as a $j$-invariant of an $A$-Drinfeld module. In this case, a component of $X_{0}(\mathfrak{p})$ can be described using a bivariate polynomial $\Phi\left(u_{1}, u_{2}\right)$ of bi-degree $(|\mathfrak{p}|+1,|\mathfrak{p}|+1)$ (that is, of degree $|\mathfrak{p}|+1$ in either of the two variables $u_{1}$ and $u_{2}$ ). Note that, for $\mathfrak{n}=1$, equation (3.3) states that

$$
g(x(1))=1+\left(q^{2}-1\right)^{-1}\left(\frac{q^{\delta}-1}{q-1} P(q)-\frac{q(q+1)}{2} \delta P(1)+\eta\right),
$$

where $\eta=-q(q-1) P(-1) / 2$ for odd $\delta$, and $\eta=0$ otherwise. As a matter of fact, this formula was stated in [6, VI.5.8] and was used as a key ingredient there to show equation (3.3). Using equation (5.1), one readily sees that $g(x(1))=0$ if $F=\mathbb{F}_{q}(T)$ and $\delta \in\{1,2,3\}$, or if $F$ is the function field of an elliptic curve and $\delta=1$. For simplicity, we assume from now on that we are in one of these situations, although the general considerations below remain valid in the general case as well. However, finding explicit equations is only possible if (the function field of) the curve $x(1)$ can be given explicitly, which is trivial if it has genus zero.

A description of $x_{0}\left(\mathfrak{p}^{2}\right)$ (a component of $X_{0}\left(\mathfrak{p}^{2}\right)$ ) can now be obtained relatively easily. A point on $X_{0}\left(\mathfrak{p}^{2}\right)$ corresponds to an isomorphism class $\left[\phi_{1}, \phi_{3}\right]$ of a pair of $\mathfrak{p}^{2}$-isogenous $A$ Drinfeld modules of rank two. Let $\mu: \phi_{1} \rightarrow \phi_{3}$ be the corresponding $\mathfrak{p}^{2}$-isogeny. Then there exists a $A$-Drinfeld module $\phi_{2}$ of rank two and p-isogenies $\lambda_{1}: \phi_{1} \rightarrow \phi_{2}$ and $\lambda_{2}: \phi_{2} \rightarrow \phi_{3}$ such that $\mu=\lambda_{2} \circ \lambda_{1}$. The isomorphism class of $\left[\phi_{i}\right]$ will correspond to a point on a component $x^{i}(1)$ of $X(1)$. This means that we can map $x_{0}\left(\mathfrak{p}^{2}\right)$ to $x^{1}(1) \times x^{2}(1) \times x^{3}(1)$. Note that both $\left[\phi_{1}, \phi_{2}\right]$ and $\left[\phi_{2}, \phi_{3}\right]$ correspond to points on $X_{0}(\mathfrak{p})$, lying on certain components, say $x_{0}^{1}(\mathfrak{p})$ and $x_{0}^{2}(\mathfrak{p})$. Using the above procedure, we can describe these two components as the zero set of polynomials $\Phi^{1}\left(u_{1}, u_{2}\right)$ and $\Phi^{2}\left(u_{2}, u_{3}\right)$, both of bi-degree $(|\mathfrak{p}|+1,|\mathfrak{p}|+1)$. This means that the image of the map from $x_{0}\left(\mathfrak{p}^{2}\right)$ to $x^{1}(1) \times x^{2}(1) \times x^{3}(1)$ is part of the zero set of the polynomials $\Phi^{1}\left(u_{1}, u_{2}\right)$ and $\Phi^{2}\left(u_{2}, u_{3}\right)$. However, this zero set turns out to be too large. The reason for this is that if $\left(\phi_{1}, \phi_{2}\right)$ and $\left(\phi_{2}, \phi_{3}\right)$ are two pairs of $\mathfrak{p}$-isogenous $A$-Drinfeld modules of rank two, with $\mathfrak{p}$-isogenies denoted by $\lambda_{1}$ and $\lambda_{2}$, then $\lambda_{2} \circ \lambda_{1}$ is either a $\mathfrak{p}^{2}$-isogeny, or has kernel isomorphic to $A / \mathfrak{p} \times A / \mathfrak{p}$. Here we used that $\mathfrak{p}$ is a prime ideal. The latter case gives rise to additional elements in the zero set of $\Phi^{1}\left(u_{1}, u_{2}\right)$ and $\Phi^{2}\left(u_{2}, u_{3}\right)$. However, this issue is rather easy to resolve: we work over the function field of $x_{0}^{1}(\mathfrak{p})$, which we can construct using 


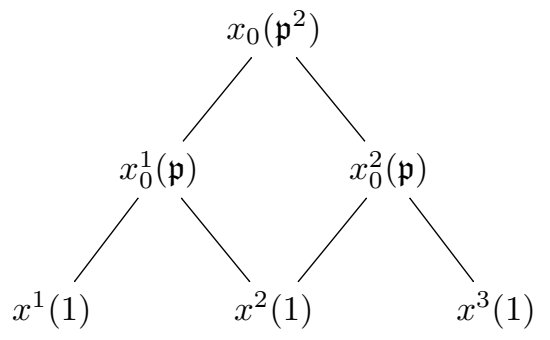

FiguRE 2. Recursive description of $x_{0}\left(\mathfrak{p}^{2}\right)$.

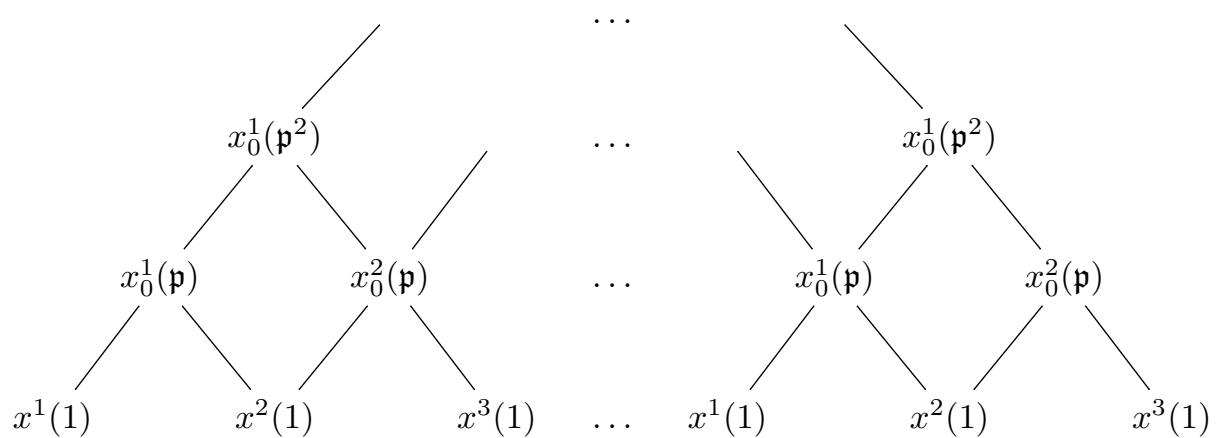

Figure 3. The pyramid of Drinfeld modular curves.

the polynomial $\Phi^{1}\left(u_{1}, u_{2}\right)$. The polynomial $\Phi^{2}\left(u_{2}, u_{3}\right)$, viewed as a univariate polynomial in $u_{3}$ and coefficients in the function field of $x_{0}^{1}(\mathfrak{p})$, has degree $|\mathfrak{p}|+1$ in $u_{3}$ while the extension degree of $X_{0}\left(\mathfrak{p}^{2}\right) / X(1)$ is $\varepsilon\left(\mathfrak{p}^{2}\right)=(|\mathfrak{p}|+1)|\mathfrak{p}|$. Then the polynomial $\Phi^{2}\left(u_{2}, u_{3}\right)$ is not absolutely irreducible and has a (for degree reasons necessarily unique) component of degree $|\mathfrak{p}|$ in $u_{3}$. This component can then be used to construct (the function field of) $x_{0}\left(\mathfrak{p}^{2}\right)$ (also see Figure 2).

Iterating this procedure gives rise to an explicit recursive description of $x_{0}\left(\mathfrak{p}^{k}\right)$ for any $k \geqslant 1$. One effectively just increases the size of the pyramid in Figures 1 and 2 . Note that, since $X(1)$ only has a finite number of absolutely irreducible components, ultimately, the same components will start to occur (see Figure 3).

In the case where $A=\mathbb{F}_{q}[T], \delta=1, \mathfrak{p}=T$ and $A$-characteristic $T-1$, explicit equations were found in [2]. In this case, all curves $X(1), X_{0}\left(\mathfrak{p}^{k}\right)$ are absolutely irreducible, so there is no need to keep track of components or to distinguish between $X_{0}\left(T^{k}\right)$ and one of its components $x_{0}\left(T^{k}\right)$. The curve $X_{0}(T)$ can be described using the Drinfeld modular polynomial $\Phi_{T}\left(u_{1}, u_{2}\right)$. However, the approach in [2] exploits the fact that the genera of the curves $X_{0}(T)$ and $X_{0}\left(T^{2}\right)$ are zero. Compared to our approach, this means that the 'pyramid' in Figure 3 starts at $X_{0}\left(\mathfrak{p}^{2}\right)$, but otherwise the recursive description is similar: the points on the curve $X_{0}\left(T^{k}\right)$ are identified with points in $X_{0}\left(T^{2}\right) \times \ldots \times X_{0}\left(T^{2}\right)$, while each of the component curves $X_{0}\left(T^{2}\right)$ can be described using a single parameter $v_{i}$. For more details see $[\mathbf{1}, \mathbf{2}]$.

\section{An new explicit example of an optimal Drinfeld modular tower}

In [1], some examples of good towers were found following the above approach, including one where the function field $F$ was the function field of an elliptic curve and $\delta=1$. More 
precisely, in the latter example in $[\mathbf{1}]$ one had $F=\mathbb{F}_{2}(X, Y)$ with $X$ transcendental over $\mathbb{F}_{2}$ and $Y^{2}+Y=X^{3}+X$, while 'infinity' was chosen to be the place at infinity of this elliptic curve, implying that $\delta=1$. The ring $A$ is then easily seen to be $\mathbb{F}_{2}[X, Y] \cong \mathbb{F}_{2}[T, S] /\left\langle S^{2}+S+T^{3}+T\right\rangle$. A description was given of the tower $X_{0}\left(\mathfrak{p}^{k}\right)$ with $\mathfrak{p}:=\langle X+1, Y+1\rangle \subset A$ and $A$-characteristic $P:=\langle X, Y\rangle$. Note that $\operatorname{deg} P=1$, since $P$ is a rational point on the elliptic curve, and ord $P=5$, since the elliptic curve has five rational points, meaning that the group of rational points is cyclic of order five. It was shown in [1] , by explicit computation, that the tower $X_{0}\left(\mathfrak{p}^{k}\right)$ (in $A$-characteristic $\langle T, S\rangle$ ) has limit at least one when the constant field is set to $\mathbb{F}_{2^{10}}$. This result is confirmed by Theorem 3.1. In this section we will describe, in a similar way to [1] , an explicit example of an optimal tower. Contrary to the example referred to above and motivated by Theorem 3.1, the choice of $A$-characteristic $P$ is now made such that ord $P=1$, implying that the resulting tower is optimal. The purpose of this example is not to give another optimal tower, but to show that an explicit description is within reach. Such a description is useful for applications in, for example, coding theory.

More precisely, we will consider the following setting:

(1) $F / \mathbb{F}_{q}:=\mathbb{F}_{2}(X, Y) / \mathbb{F}_{2}$, where $Y^{2}+X Y+X^{2}=X$ and $X$ is transcendental over $\mathbb{F}_{2}$;

(2) $A:=\mathbb{F}_{2}[X, Y]$, implying $\delta=2$; and

(3) the $A$-characteristic $P$ is the principal prime ideal $\left\langle X^{2}+X+1\right\rangle \subset A$.

Note that the function field $F$ has genus zero, implying that the L-polynomial $P(t)$ occurring in the zeta function of $F$ is simply $P(t)=1$. Therefore the curve $X(1)$ has $\delta P(1)=2$ absolutely irreducible components, say $x^{1}(1)$ and $x^{2}(1)$, both of genus zero according to equation (5.1). Since, for the given choice of $P$, we have ord $P=1$ (since $P$ is a principal ideal) and $\operatorname{deg} P=4$, Theorem 3.1 implies that, for any choice of prime ideal $\mathfrak{p} \subset A$ coprime with the $A$-characteristic $P$, the limit of the resulting family of curves $\left(X_{0}\left(\mathfrak{p}^{k}\right)\right)_{k}$, when defined over the finite field $\mathbb{F}_{2^{8}}$, equals $\sqrt{2^{8}}-1=15$. In other words, the resulting family of curves is optimal over $\mathbb{F}_{2^{8}}$.

We start by indicating how to describe $A$-Drinfeld modules explicitly. An $A$-Drinfeld module of rank two is symbolically determined by

$$
\begin{aligned}
& \phi_{X}=g_{0} \tau^{4}+g_{1} \tau^{3}+g_{2} \tau^{2}+g_{3} \tau+\iota(X), \\
& \phi_{Y}=h_{0} \tau^{4}+h_{1} \tau^{3}+h_{2} \tau^{2}+h_{3} \tau+\iota(Y) .
\end{aligned}
$$

Since we have chosen the principal prime ideal $\left\langle X^{2}+X+1\right\rangle$ as $A$-characteristic, we have $\iota(X)^{2}+\iota(X)+1=0$ and, using the equation of the curve, $\iota(Y)^{2}+\iota(X) \iota(Y)+\iota(X)^{2}=\iota(X)$. For convenience we will write

$$
x:=\iota(X) \quad \text { and } \quad y:=\iota(Y) .
$$

We see that $x=\iota(X) \in \mathbb{F}_{4}$ and $y=\iota(Y) \in \mathbb{F}_{16}$. The remaining coefficients also satisfy several algebraic relations, stemming from the fact that $\phi_{X} \phi_{Y}=\phi_{Y} \phi_{X}$ and $\phi_{Y^{2}+X Y+X^{2}-X}=0$. Indeed, any choice of $g_{0}, \ldots, h_{3}$ satisfying these relations gives rise to a Drinfeld module. The equation $\phi_{X} \phi_{Y}=\phi_{Y} \phi_{X}$, implies that

$$
\begin{aligned}
& g_{0} h_{0}^{q^{4}}=h_{0} g_{0}^{q^{4}} \\
& g_{0} h_{1}^{q^{4}}+g_{1} h_{0}^{q^{3}}=h_{0} g_{1}^{q^{4}}+h_{1} g_{0}^{q^{3}} \\
& g_{0} h_{2}^{q^{4}}+g_{1} h_{1}^{q^{3}}+g_{2} h_{0}^{q^{2}}=h_{0} g_{2}^{q^{4}}+h_{1} g_{1}^{q^{3}}+h_{2} g_{0}^{q^{2}} \\
& g_{0} h_{3}^{q^{4}}+g_{1} h_{2}^{q^{3}}+g_{2} h_{1}^{q^{2}}+g_{3} h_{0}^{q}=h_{0} g_{3}^{q^{4}}+h_{1} g_{2}^{q^{3}}+h_{2} g_{1}^{q^{2}}+h_{3} g_{0}^{q} \\
& g_{1} h_{3}^{q^{3}}+g_{2} h_{2}^{q^{2}}+g_{3} h_{1}^{q}+x h_{0}=h_{0} x^{q^{4}}+h_{1} g_{3}^{q^{3}}+h_{2} g_{2}^{q^{2}}+h_{3} g_{1}^{q} \\
& g_{1} y^{q^{3}}+g_{2} h_{3}^{q^{2}}+g_{3} h_{2}^{q}+x h_{1}=h_{1} x^{q^{3}}+h_{2} g_{3}^{q^{2}}+h_{3} g_{2}^{q}+y g_{1}
\end{aligned}
$$




$$
\begin{aligned}
g_{2} y^{q^{2}}+g_{3} h_{3}^{q}+x h_{2} & =h_{2} x^{q^{2}}+h_{3} g_{3}^{q}+y g_{2} \\
g_{3} y^{q}+x h_{3} & =h_{3} x^{q}+y g_{3} .
\end{aligned}
$$

Note that, throughout this section, we assume that $q=2$. Similarly, the equation $\phi_{Y^{2}+X Y+X^{2}-X}=0$ gives rise to algebraic relations. From equations (6.6)-(6.8), one sees that the three variables $g_{3}, g_{2}, g_{1}$ can be expressed in the three variables $h_{3}, h_{2}, h_{1}$. After eliminating $g_{1}, g_{2}, g_{3}$ in this way, equations (6.2)-(6.5) give rise to pairs of polynomials in $h_{1}$. These polynomials turn out to have a very special form: they are linearized polynomials in $h_{1}$ plus a constant term. Therefore we can use the $q$-linearized variant of the Euclidean algorithm to eliminate the variable $h_{1}$ very efficiently, so avoiding a lengthy Groebner basis computation. Finally, we may use equation (6.1) to normalize the leading coefficients $g_{0}$ and $h_{0}$ by putting $h_{0}=1$ and choosing $g_{0} \in \mathbb{F}_{4}$ such that $g_{0}^{2}+g_{0}+1=0$. We are then left with an explicit algebraic equation relating $h_{2}$ and $h_{3}$, say $f\left(h_{2}, h_{3}\right)=0$, with coefficients in $\mathbb{F}_{16}$. The equation is a bit lengthy, but we state it for the sake of completeness.

$$
\begin{aligned}
& f\left(h_{2}, h_{3}\right)=h_{2}^{30}+(x y+x) h_{2}^{29} h_{3}^{3}+(y+x) h_{2}^{27} h_{3}^{9}+(x y+1) h_{2}^{26} h_{3}^{12}+(y+1) h_{2}^{25} \\
& +(x y+x) h_{2}^{24} h_{3}^{18}+\left(x^{2} y+x^{2}\right) h_{2}^{24} h_{3}^{3}+y h_{2}^{23} h_{3}^{21}+\left(x^{2} y+1\right) h_{2}^{23} h_{3}^{6}+x^{2} y h_{2}^{22} h_{3}^{9} \\
& +(x y+1) h_{2}^{21} h_{3}^{27}+\left(x^{2} y+x\right) h_{2}^{21} h_{3}^{12}+h_{2}^{20} h_{3}^{30}+(y+1) h_{2}^{20} h_{3}^{15}+(x y+1) h_{2}^{20} \\
& +\left(x^{2} y+x^{2}\right) h_{2}^{19} h_{3}^{18}+y h_{2}^{18} h_{3}^{36}+(x y+x) h_{2}^{18} h_{3}^{6}+(y+x) h_{2}^{17} h_{3}^{39}+\left(y+x^{2}\right) h_{2}^{17} h_{3}^{24} \\
& +x h_{2}^{17} h_{3}^{9}+\left(x^{2} y+1\right) h_{2}^{16} h_{3}^{27}+x y h_{2}^{16} h_{3}^{12}+h_{2}^{15} h_{3}^{45}+(y+1) h_{2}^{15} h_{3}^{30}+x y h_{2}^{15} h_{3}^{15} \\
& +(y+x) h_{2}^{15}+\left(x^{2} y+x^{2}\right) h_{2}^{14} h_{3}^{33}+(y+1) h_{2}^{14} h_{3}^{18}+h_{2}^{14} h_{3}^{3}+y h_{2}^{13} h_{3}^{51}+x y h_{2}^{13} h_{3}^{36} \\
& +x h_{2}^{13} h_{3}^{21}+(x y+x) h_{2}^{13} h_{3}^{6}+(y+x) h_{2}^{12} h_{3}^{54}+x^{2} y h_{2}^{12} h_{3}^{39}+\left(x^{2} y+x\right) h_{2}^{12} h_{3}^{9} \\
& +\left(x^{2} y+x\right) h_{2}^{11} h_{3}^{42}+\left(y+x^{2}\right) h_{2}^{11} h_{3}^{27}+x h_{2}^{11} h_{3}^{12}+h_{2}^{10} h_{3}^{60}+\left(y+x^{2}\right) h_{2}^{10} h_{3}^{45}+x h_{2}^{10} h_{3}^{30} \\
& +(y+x) h_{2}^{10} h_{3}^{15}+(x y+1) h_{2}^{10}+(x y+x) h_{2}^{9} h_{3}^{63}+x^{2} y h_{2}^{9} h_{3}^{48}+(x y+x) h_{2}^{9} h_{3}^{33} \\
& +(x y+1) h_{2}^{9} h_{3}^{18}+(x y+x) h_{2}^{9} h_{3}^{3}+x y h_{2}^{8} h_{3}^{51}+\left(x^{2} y+x\right) h_{2}^{8} h_{3}^{36}+(x y+x) h_{2}^{8} h_{3}^{21} \\
& +(y+x) h_{2}^{8} h_{3}^{6}+(y+x) h_{2}^{7} h_{3}^{69}+\left(y+x^{2}\right) h_{2}^{7} h_{3}^{54}+\left(x^{2} y+1\right) h_{2}^{7} h_{3}^{39}+(x y+1) h_{2}^{7} h_{3}^{24} \\
& +x h_{2}^{7} h_{3}^{9}+(x y+1) h_{2}^{6} h_{3}^{72}+x y h_{2}^{6} h_{3}^{42}+(x y+1) h_{2}^{6} h_{3}^{27}+\left(y+x^{2}\right) h_{2}^{6} h_{3}^{12}+x h_{2}^{5} h_{3}^{60} \\
& +(x y+1) h_{2}^{5} h_{3}^{45}+h_{2}^{5} h_{3}^{30}+\left(x y+x^{2}\right) h_{2}^{5} h_{3}^{15}+(y+1) h_{2}^{5}+(x y+x) h_{2}^{4} h_{3}^{78}+y h_{2}^{4} h_{3}^{48} \\
& +\left(x^{2} y+x\right) h_{2}^{4} h_{3}^{33}+(x y+x) h_{2}^{4} h_{3}^{18}+x^{2} h_{2}^{4} h_{3}^{3}+y h_{2}^{3} h_{3}^{81}+x y h_{2}^{3} h_{3}^{66}+x h_{2}^{3} h_{3}^{51} \\
& +\left(x^{2} y+x^{2}\right) h_{2}^{3} h_{3}^{36}+x y h_{2}^{3} h_{3}^{21}+\left(x y+x^{2}\right) h_{2}^{2} h_{3}^{69}+(y+x) h_{2}^{2} h_{3}^{54}+(y+1) h_{2}^{2} h_{3}^{39} \\
& +(y+x) h_{2}^{2} h_{3}^{24}+\left(y+x^{2}\right) h_{2}^{2} h_{3}^{9}+(x y+1) h_{2} h_{3}^{87}+h_{2} h_{3}^{57}+x^{2} y h_{2} h_{3}^{42}+\left(x^{2} y+x^{2}\right) h_{2} h_{3}^{27} \\
& +\left(x^{2} y+1\right) h_{2} h_{3}^{12}+h_{3}^{90}+x h_{3}^{75}+h_{3}^{60}+x^{2} h_{3}^{45}+x^{2} h_{3}^{30}+1 \text {. }
\end{aligned}
$$

This equation does not describe the curve $X(1)$, since we have not yet considered isomorphism classes of $A$-Drinfeld modules. Therefore, let $\psi$ be another $A$-Drinfeld module, with the same $A$-characteristic and normalized in the same way as $\phi$, defined by

$$
\begin{aligned}
& \psi_{X}=l_{0} \tau^{4}+l_{1} \tau^{3}+l_{2} \tau^{2}+l_{3} \tau+\iota(X), \\
& \psi_{Y}=t_{0} \tau^{4}+t_{1} \tau^{3}+t_{2} \tau^{2}+t_{3} \tau+\iota(Y)
\end{aligned}
$$

An isomorphism between $\phi$ and $\psi$ is a non-zero constant $c$ such that $c \phi=\psi c$. By considering, for example, the leading coefficient of $c \phi_{Y}=\psi_{Y} c$ we get $c^{q^{4}-1}=1$, implying that

$$
t_{1}^{(q+1)\left(q^{2}+1\right)}=h_{1}^{(q+1)\left(q^{2}+1\right)}, \quad t_{2}^{q^{2}+1}=h_{2}^{q^{2}+1}, \quad t_{3}^{(q+1)\left(q^{2}+1\right)}=h_{3}^{(q+1)\left(q^{2}+1\right)} .
$$

In other words, the quantities $h_{1}^{(q+1)\left(q^{2}+1\right)}, h_{2}^{q^{2}+1}$ and $h_{3}^{(q+1)\left(q^{2}+1\right)}$ (and, similarly, $g_{11}:=$ $g_{1}^{(q+1)\left(q^{2}+1\right)}, g_{22}:=g_{2}^{q^{2}+1}$ and $\left.g_{33}:=g_{3}^{(q+1)\left(q^{2}+1\right)}\right)$ are invariants of $A$-Drinfeld modules.

Putting $h_{22}:=h_{2}^{q^{2}+1}$ and $h_{33}:=h_{3}^{(q+1)\left(q^{2}+1\right)}$, the previously found equation $f\left(h_{2}, h_{3}\right)=0$, relating $h_{2}$ and $h_{3}$, gives rise to a relation $p\left(h_{22}, h_{33}\right)=0$. One simply uses the relations 
$f\left(h_{2}, h_{3}\right), h_{2}^{q^{2}+1}-h_{22}$ and $h_{3}^{(q+1)\left(q^{2}+1\right)}-h_{33}$ and eliminates the variables $h_{2}$ and $h_{3}$, using a Groebner basis computation. The resulting relation $p\left(h_{22}, h_{33}\right)=0$ then defines the Drinfeld modular curve $X(1)$. This is not immediately clear, since, strictly speaking, we only can be certain that the function field generated by $h_{22}$ and $h_{33}$ is a subfield of the function field of $X(1)$. However, again using a computer to perform a Groebner basis computation, one can show that this subfield already contains the remaining invariants $h_{11}, g_{11}, g_{22}$, and $g_{33}$. At first sight, it might look as if $\mathbb{F}_{16}\left(h_{22}, h_{33}\right)$ has index 75 in $\mathbb{F}_{16}\left(h_{2}, h_{3}\right)$. With a computer, it can be verified that $h_{2}$ can be expressed in $h_{22}$ and $h_{3}$, implying that the index of $\mathbb{F}_{16}\left(h_{22}, h_{33}\right)$ in $\mathbb{F}_{16}\left(h_{2}, h_{3}\right)$ is, in fact, only 15 , in accordance with the number of possible choices of the isomorphism $c$ mentioned before equation (6.9).

So far, we have computed an explicit model for the curve $X(1)$. The theory implies that this curve has two components. Indeed, according to this prediction, the bivariate polynomial $p(t, s)$ is not absolutely irreducible, but has two absolutely irreducible factors, say $p^{1}(t, s)$ and $p^{2}(t, s)$, which turn out to have coefficients in $\mathbb{F}_{16}$. These factors define the curves that were previously denoted by $x^{1}(1)$ and $x^{2}(1)$.

To start a recursive description of a tower of function fields, we choose one of the components, say the one defined by $p^{1}\left(h_{22}, h_{33}\right)=0$ for the component denoted by $x^{1}(1)$. Since this curve has genus zero by equation (5.1), its function field is rational and can be described using a parameter $u$, so $\mathbb{F}_{16}\left(h_{22}, h_{33}\right)=\mathbb{F}_{16}(u)$.

To describe a tower, as in the previous section, we need to choose a prime ideal $\mathfrak{p}$. In this section, we choose $\mathfrak{p}=\langle X, Y\rangle \subset A$, which is coprime with the $A$-characteristic. Since $\operatorname{deg} \mathfrak{p}=1$, a p-isogeny $\lambda$ between $\phi$ and $\psi$ is of the form $\tau-a$. From the isogeny property $\lambda \phi_{Y}=\psi_{Y} \lambda$ and using as before $x:=\iota(X)$ and $y:=\iota(Y)$, we get

$$
\begin{aligned}
& t_{3}=a^{-q}\left(y-y^{q}+a h_{3}\right), \\
& t_{2}=a^{-q^{2}} t_{3}+a^{1-q^{2}} h_{2}-a^{-q^{2}} h_{3}^{q} .
\end{aligned}
$$

A direct verification shows that if we set $t_{33}=t_{3}^{(q+1)\left(q^{2}+1\right)}$ and $t_{22}=t_{2}^{q^{2}+1}$, then $t_{33}, t_{22}$ satisfy $p^{2}\left(t_{22}, t_{33}\right)=0$. In other words, the isogeny maps the component $x^{1}(1)$ of $X(1)$ to the other component $x^{2}(1)$. Similar to the uniformizing parameter $u$ of $x^{1}(1)$, one can find a uniformizing parameter $v$ of $x^{2}(1)$. Using the above isogeny relation, we can compute $\Phi^{1}(u, v)=0$, defining $x_{0}^{1}(\mathfrak{p})$ as in Figure 4. Similarly, starting with the component $x^{2}(1)$, one finds the relation $\Phi^{2}(v, w)=0$ defining $x_{0}^{2}(\mathfrak{p})$. Explicitly, one obtains

$$
\begin{aligned}
\Phi^{1}(u, v)= & \left(u+\left(x^{2} y+1\right)\right) v^{3} \\
& +\left(y u^{3}+(x y+1) u^{2}+x^{2} y u+(x y+x)\right) v^{2} \\
& +\left(\left(y+x^{2}\right) u^{2}+\left(x^{2} y+1\right) u+(x y+1)\right) v \\
& +(y+1) u^{3}+x u^{2}+y u+x^{2} y+x^{2}, \\
\Phi^{2}(v, w)= & (v+x y) w^{3} \\
& +\left((y+x) v^{3}+x^{2} y v^{2}+x y v+1\right) w^{2} \\
& +\left((y+1) v^{2}+v+(y+1)\right) w \\
& +\left(x^{2} y+x\right) v^{3}+(y+x) v^{2}+(x y+1) v+x y .
\end{aligned}
$$

Now we can construct the tower of function fields $\mathcal{F}=\left(F_{0} \subset F_{1} \subset \ldots\right)$ corresponding to the modular tower $\left(x_{0}\left(\mathfrak{p}^{k}\right)\right)_{k}$ by

(1) $F_{0}=\mathbb{F}_{16}\left(u_{0}\right)$;

(2) $F_{1}=F_{0}\left(u_{1}\right)$ with $\Phi^{1}\left(u_{0}, u_{1}\right)=0$; and

(3) $F_{k}=F_{k-1}\left(u_{k}\right)$, where $\Phi^{1}\left(u_{k-1}, u_{k}\right)=0$ if $k$ odd, and $\Phi^{2}\left(u_{k-1}, u_{k}\right)=0$ otherwise. 


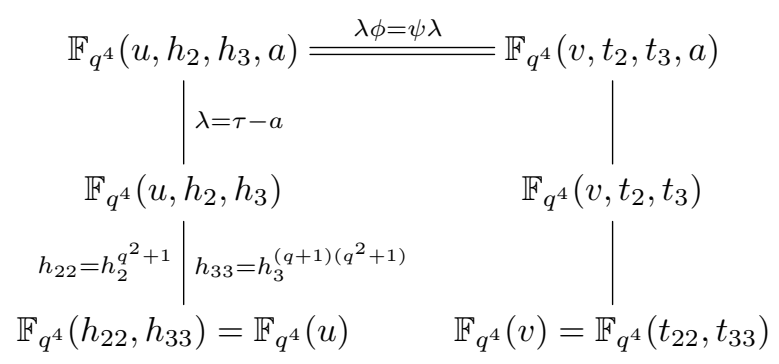

FIGURE 4. Defining $x_{0}^{1}(\mathfrak{p})$ explicitly by $\Phi^{1}(u, v)=0$.

As remarked in $\S 5$, for $k>1$, the equations $\Phi^{i}\left(u_{k-1}, u_{k}\right)=0$ give rise to two possible factors: the first of degree one in $u_{k}$ and the second of degree $|\mathfrak{p}|=q=2$. The factor of degree two should be chosen when defining the tower.

\section{Conclusion}

In this paper we give a recursive description of (reductions of) Drinfeld modular towers for any possible base ring $A$ as well as a lower bound for the limit of such towers. It turns out that good reductions of Drinfeld modular towers are always good, when defined over the proper constant field, but not always optimal. The theory presented here fully explains the behaviour of a Drinfeld modular tower given in [1]. Furthermore, we give an explicit recursive description of an optimal Drinfeld tower over $\mathbb{F}_{16}$ that has not been previously considered in the literature. In addition, this demonstrates that explicit descriptions of Drinfeld modular towers are not restricted to the case in which the base ring $A$ is the polynomial ring.

Acknowledgements. The authors would like to thank the anonymous referee for helpful suggestions and comments, that helped to improve the paper. The last two authors gratefully acknowledge the support from the Danish National Research Foundation and the National Science Foundation of China (Grant No. 11061130539) for the Danish-Chinese Center for Applications of Algebraic Geometry in Coding Theory and Cryptography as well as the support from The Danish Council for Independent Research (Grant No. DFF-4002-00367). The first author is supported by Tubitak Proj. No. 112T233.

\section{References}

1. A. Bassa, P. Beelen and N. Nguyen, 'Good towers of function fields', Algebraic curves and finite fields, Radon Series on Computational and Applied Mathematics (Walter de Gruyter, Berlin/Boston, 2014) 23-40.

2. N. D. ElkiEs, 'Explicit modular towers', Proceedings of the Thirty-Fifth [1997] Annual Allerton Conference on Communication, Control and Computing (University of Illinois at Urbana-Champaign, 1998) 23-32.

3. N. D. Elkies, 'Explicit towers of Drinfeld modular curves', European congress of mathematics, Progress In Mathematics 202 (Birkhäuser, Basel, 2001) 189-198.

4. A. Garcia and H. Stichtenoth, 'On the asymptotic behaviour of some towers of function fields over finite fields', J. Number Theory 61 (1996) no. 2, 248-273.

5. E.-U. Gekeler, 'Drinfeld-Moduln und modulare Formen über rationalen Funktionenkörpern', Bonner mathematische Schriften (Mathematischen Institut der Universität Bonn, 1979).

6. E.-U. Gekeler, Drinfeld modular curves, Lecture Notes in Mathematics 1231 (Springer, Heidelberg, 1986).

7. E.-U. Gekeler, 'Sur la géométrie de certaines algèbres de quaternions', J. Théor. Nombres Bordeaux (2) 2 (1990) no. $1,143-153$. 
8. E.-U. GeKeler, 'Asymptotically optimal towers of curves over finite fields', Algebra, arithmetic and geometry with applications (Springer, Heidelberg, 2004) 325-336.

9. D. M. Goldschmid, 'Algebraic functions and projective curves', Graduate Texts in Mathematics 215 (Springer, New York, 2003).

10. V. D. Goppa, 'Codes on algebraic curves', Sov. Math. Dokl. 24 (1981) no. 1, 170-172.

11. D. Goss, Basic structures of function field arithmetic (Springer, Heidelberg, 1996).

12. Y. IharA, 'Some remarks on the number of rational points of algebraic curves over finite fields', J. Fac. Sci. Univ. Tokyo 1A 28 (1982) 721-724.

13. A. Schweizer, 'Hyperelliptic Drinfeld modular curves', Drinfeld modules, modular schemes and applications (World Scientific, Singapore, 1997) 330-343.

14. J.-P. Serre, 'Sur le nombre des points rationnels d'une courbe algébrique sur un corps fini', C. R. Acad. Sci. Paris 296 (1983) 397-402.

15. L. TAelman, 'Drinfeld modular curves have many points', Preprint, 2006, arXiv:math/0602157 [math.AG].

16. M. A. Tsfasman, S. G. VlÂdut and Th. Zink, 'Modular curves, Shimura curves, and Goppa codes, better than Varshamov-Gilbert bound', Math. Nachr. 109 (1982) no. 1, 21-28.

17. S. G. Vhâdut and V. G. Drinfel'D, 'Number of points of an algebraic curve', Funct. Anal. Appl. 17 (1983) no. 1, 53-54.

Alp Bassa

Boğaziçi University

Faculty of Arts and Sciences

Department of Mathematics

34342 Bebek

Istanbul

Turkey

alp.bassa@boun.edu.tr
Peter Beelen

Technical University of Denmark

Department of Applied Mathematics and

Computer Science

Matematiktorvet 303B

2800 Kgs. Lyngby

Denmark

pabe@dtu.dk

\author{
Nhut Nguyen \\ Technical University of Denmark \\ Department of Applied Mathematics and \\ Computer Science \\ Matematiktorvet 303B \\ 2800 Kgs. Lyngby \\ Denmark \\ nhngu@dtu.dk
}

